Article

\title{
Cauchy Principal Value Contour Integral with Applications
}

\author{
Matilde Legua ${ }^{1}$ and Luis M. Sánchez-Ruiz ${ }^{2, *}$ \\ 1 EINA-Departamento de Matemática Aplicada, Universidad de Zaragoza, Zaragoza 50018, Spain; \\ mlegua@unizar.es \\ 2 CITG-Departamento de Matemática Aplicada, Universitat Politècnica de València, Valencia 46022, Spain \\ * Correspondence: lmsr@mat.upv.es; Tel.: +34-963-877-665
}

Academic Editors: Carlo Cattani and Kevin Knuth

Received: 28 March 2017; Accepted: 3 May 2017; Published: 10 May 2017

\begin{abstract}
Cauchy principal value is a standard method applied in mathematical applications by which an improper, and possibly divergent, integral is measured in a balanced way around singularities or at infinity. On the other hand, entropy prediction of systems behavior from a thermodynamic perspective commonly involves contour integrals. With the aim of facilitating the calculus of such integrals in this entropic scenario, we revisit the generalization of Cauchy principal value to complex contour integral, formalize its definition and - by using residue theory techniques—provide an useful way to evaluate them.
\end{abstract}

Keywords: Cauchy principal value; contour integral; entropy as measurement; information extraction; thermodynamics; aerodynamics

\section{Introduction}

Continuous intrinsic entropy treatment requires the assistance of measure theory. This involves adequate integrals intended to evaluate the unexpected uncertainty, indeed this is the case when differential entropy is studied [1-5] in which case improper integrals are commonly used. A number of analytical and numerical methods have been developed such as the Glauert and Goldstein methods developed to study improper integrals that appear in this setting, [6-11], which are relevant in aerodynamics. In fact, aerodynamic entropy study focuses on the overall performance neglecting singularities that enjoy some symmetric entropic phenomena such as turbulence on wing or rotor blades and for this Cauchy principal values are commonly used [12-14]. Cauchy principal value contour integrals have recently arisen inspired by the real case definition, [15]. In this paper, we revisit this extension, formalize its definition, and provide an useful method for its calculus by residue theory and apply it to some improper integrals involving trigonometric functions including the Glauert method used in thermodynamic study of a 2D wing section.

The article is organized as follows. Previous related work and background is detailed in Section 2. In Section 3, we study the Cauchy principal value contour integral in the complex field and in Section 4 we relate it to the classical residue theory of complex variables. Applications to quadrature are provided in Section 5. Section 6 provides discussion, and the conclusions drawn appear in Section 7.

\section{Background}

Improper integral of a real function $f$ between reals $a$ and $b$ when $f$ has got a vertical asymptote at some extreme point, say $b$ - the other extreme case is analogous-, (or if $b$ is $\infty$ ) is defined by taking adequate limits [12],

$$
\lim _{c \rightarrow b^{-}} \int_{a}^{c} f(x) d x\left(\text { or } \lim _{b \rightarrow+\infty} \int_{a}^{b} f(x) d x\right)
$$


This limit provides the value of Lebesgue integrals and, even in some cases when the latter does not exist, as for example it happens with $\int_{[0,+\infty[} \frac{\sin (x)}{x} d x$ that does not exist according to measure theory. However $\int_{0}^{+\infty} \frac{\sin (x)}{x} d x=\frac{\pi}{2}$ is an example of an improper integral classically evaluated by using residue theory, cf. [16-18].

Improper integrals with more than one singularity in play (including infinite endpoints) are convergent if, splitting the interval of integration in such a way that in each subinterval we consider one limit at a time, each of the split integrals is convergent. On the other hand, the Cauchy principal value arises when the singularities are handled in a balanced way, e.g., if $a<c<b$ and $f$ has got a vertical asymptote at $c$ the principal value of $\int_{a}^{b} f(x) d x$ is

$$
\mathrm{PV} \int_{a}^{b} f(x) d x=\lim _{\epsilon \rightarrow 0^{+}}\left(\int_{a}^{c-\epsilon} f(x) d x+\int_{c+\epsilon}^{b} f(x) d x\right) .
$$

and, when both endpoints are infinite, the principal value is calculated as

$$
\mathrm{PV} \int_{-\infty}^{+\infty} f(x) d x=\lim _{R \rightarrow+\infty}\left(\int_{-R}^{R} f(x) d x\right) .
$$

Clearly, if an improper integral is convergent, it converges to its principal value but the latter may exist though the corresponding improper integral does not.

The integral of a complex function $f$ of real variable $f:[a, b] \rightarrow \mathbb{C}$, is defined by splitting the real and imaginary part, cf. [16-18],

$$
\int_{a}^{b} f(x) d x=\int_{a}^{b} \operatorname{Re}(f(x)) d x+\mathrm{i} \int_{a}^{b} \operatorname{Im}(f(x)) d x .
$$

Here improper integrals may appear, e.g., in the very definition of Fourier transform, given by $F[f(x)](\lambda)=\int_{-\infty}^{+\infty} \exp (-i \lambda t) f(t) d t$, or Laplace transform, $\mathcal{L}[f(x)](\mathrm{s})=\int_{0}^{+\infty} \exp (-s x) f(x) d x$ where the parameter $s$ is complex. Cauchy principal values are subject to being considered in these complex integrals, too, cf. [18].

Contour integrals rely on the path, also called contour, $\gamma$ on which it is defined, i.e., a function $\gamma:[a, b] \rightarrow \mathbb{C}$ of class $C^{1}$ which is regular, that is with $\gamma^{\prime}(t) \neq 0$ in $[a, b]$. Then, if $f$ is a complex function defined on $\gamma[a, b]$, the contour integral of $f$ along $\gamma, \int_{\gamma} f(z) d z$, is defined by the complex integral of real variable $\int_{a}^{b} f(\gamma(t)) \gamma^{\prime}(t) d t$ and expressed as the sum of two line integrals by splitting the real and imaginary part, [16-18]. Contour integrals are easily extended to piecewise paths $\gamma$, i.e., when $\gamma:[a, b] \rightarrow \mathbb{C}$ is continuous and there is some partition $a=t_{0}<t_{1}<\ldots<t_{n}=b$ so that the restriction $\gamma_{i}$ of $\gamma$ to each $\left[t_{i}, t_{i+1}\right]$ is of class $C^{1}$ and regular, as a sum of the corresponding contour integrals of $f$ along $\gamma_{i}$. We will eventually represent a contour integral $\int_{\gamma} f(z) d z$ as $\int_{\gamma[a, b]} f(z) d z$ and identify $\gamma$ with $\gamma[a, b]$ as far as there is no misunderstanding.

Contour integration appears in entropy study and is the backbone of residue theory that, in turn, finds many well-known applications to evaluate some improper integrals and, a fortiori, to calculate Fourier, Laplace, and $\mathrm{Z}$ transforms, all of them basic tools in operational calculus and signal analysis, cf. [16-18].

\section{Cauchy Principal Value Contour Integral}

Contour integral $\int_{\gamma} f(z) d z$ requires the function $f$ to be defined on the path $\gamma$. Nevertheless, an analog to the real Cauchy integral principal values was considered in [15] in case of unboundedness of $f$ at some intermediate point $c$ of $\gamma$, by skipping its part located inside the circumference of radius $\varepsilon>0$ centered at $c$, and then taking limits when $\varepsilon \rightarrow 0$. In this section, we revisit this idea by including adequate definitions and show that residue theory may be applied. Firstly, we formalize the equivalent 
to the real improper integrals corresponding to some unbounded function at an extreme of its interval of definition.

Definition 1. Let $\gamma:[a, b] \rightarrow \mathbb{C}$ be a path and let $f$ be a function defined on $\gamma$ except at the extreme point $\gamma(b)$ (resp. $\gamma(a))$, the improper contour integral of f along $\gamma$ is defined by the following limit, if it exists,

$$
\int_{\gamma} f(z) d z=\lim _{c \rightarrow b^{-}} \int_{a}^{c} f(\gamma(t)) \gamma^{\prime}(t) d t \quad\left(\text { resp. } \lim _{c \rightarrow a^{+}} \int_{c}^{b} f(\gamma(t)) \gamma^{\prime}(t) d t\right) .
$$

It is pretty clear that Definition 1 includes the case of complex improper integrals of real variable corresponding to unboundedness at the extreme of an interval by taking $\gamma(t)=t$.

Definition 2. Let $\gamma:[a, b] \rightarrow \mathbb{C}$ be a piecewise path corresponding to a partition $\left\{t_{i}, 0 \leq i \leq n\right\}$ of $[a, b]$, so that the restriction $\gamma_{i}$ of $\gamma$ to each $\left[t_{i}, t_{i+1}\right]$ is of class $C^{1}$ and regular, and let $f$ be a function defined on $\gamma$ except at the points $\gamma\left(t_{i}\right)$, the improper contour integral of falong $\gamma$ is defined by the following sum, if it exists,

$$
\int_{\gamma} f(z) d z=\sum_{i=0}^{n-1} \int_{\gamma_{i}} f(z) d z
$$

Definition 2 includes the improper real integrals corresponding to unboundedness at intermediate points of the interval of integration as a particular case by considering $\gamma(t)=t$ and $t_{i}$, the points where side limits are to be taken.

The following definition aims at considering the Cauchy principal value of an improper contour integral along a piecewise path $\gamma$. In order to simplify notation in the definition, we consider only one intermediate point $c$ so that the function is unbounded at some neighborhood of $\gamma(c)$. The idea is to obtain two adequate paths from the original one, so that one of them ends at the same distance $r$ as the other one starts respect to $\gamma(c)$ and then take limits as $r$ tends to 0 .

Definition 3. Let $\gamma:[a, b] \rightarrow \mathbb{C}$ be a piecewise path and let $f$ be a function defined on $\gamma[a, b]$ except at $\gamma(c)$, $a<c<b$. Set $d_{1, c}(r)=\inf \{t \in[a, c]:|\gamma(c)-\gamma(t)|<r\}$ and $d_{2, c}(r)=\sup \{t \in[c, b]:|\gamma(c)-\gamma(t)|<r\}$, and represent by $\gamma_{d 1, r}(c)\left(\right.$ resp. $\left.\gamma_{d 2, r}(c)\right)$ the restriction of $\gamma$ to $\left[a, d_{1, c}(r)\right]\left(\right.$ resp. $\left.\left[d_{2, c}(r), b\right]\right)$. We will we call the Cauchy principal value contour integral of $f$ along $\gamma$ to the following limit, if it exists,

$$
\operatorname{PV} \int_{\gamma} f(z) d z=\lim _{r \rightarrow 0^{+}}\left(\int_{\gamma_{d 1, r}(c)} f(z) d z+\int_{\gamma_{d 2, r}(c)} f(z) d z\right) .
$$

Definition 3 is consistent as $\gamma$ is continuous and regular. Definition 3 generates the Cauchy improper real integral in the same way as Definition 2 by considering $\gamma(t)=t$.

As in the real case, if an improper contour integral is convergent, it converges to its principal value but the latter may exist though the corresponding improper integral does not.

If there are several unboundedness points of $f$ along $\gamma$, the above process is to be repeated at each of them as done in the following example.

Example 1. Find $P V \int_{\gamma} f(z) d z$ for $f(z)=\frac{1}{1+z^{2}}$ and $\gamma(t)=i t, t \in[a, b]$ with $a<-1,1<b$. The function $f$ is unbounded at any neighborhood of $\pm i$ and in this case the improper contour integral does not exist. By splitting the function $f(z)=\frac{-1 /(2 i)}{z+i}+\frac{1 /(2 i)}{z-i}$, considering the sum of the integral contour of $f$ along the paths obtained by restricting $\gamma$ to

$$
[a,-1-r],[-1+r, 1-r],[1+r, b],
$$

and taking limits when $r$ tends to $0^{+}$, we obtain $P V \int_{\gamma} f(z) d z=\frac{1}{2} \ln \left(\frac{b+1}{-1-a} \frac{1-a}{b-1}\right)$.

Definition 3 is inspired and formalizes the idea exposed in [15]. It disregards the length of the path before and after the point where the function is unbounded. If one wishes to deal with this length 
of the arc as an intrinsic issue in order to define the Cauchy principal contour integral, then we propose the following natural method. Given the path $\gamma:[a, b] \rightarrow \mathbb{C}$, for $a<u<v<b$ we will write down $\mathrm{L}(\gamma[u, v])$ to denote the length of $\gamma[u, v]$, which is known to be given by $\int_{u}^{v}\left|\gamma^{\prime}(t)\right| d t$.

Definition 4. Let $\gamma:[a, b] \rightarrow \mathbb{C}$ be a piecewise path and let $f$ be a function defined on $\gamma[a, b]$ except at $\gamma(c), a<c<b$. Set $l_{1, c}(r)=\inf \{t \in[a, c]: L(\gamma[t, c])<r\}$ and $l_{2, c}(r)=\sup \{t \in[c, b]: L(\gamma[c, t])<r\}$, and represent by $\gamma_{l 1, r}(c)$ (resp. $\gamma_{l 2, r}(c)$ ) the restriction of $\gamma$ to $\left[a, l_{1, c}(r)\right]\left(\right.$ resp. $\left.\left[l_{2, c}(r), b\right]\right)$. We will we call the Cauchy principal value contour integral of $f$ along $\gamma$ with respect to the arc length to the following limit, if it exists,

$$
\operatorname{PVA} \int_{\gamma} f(z) d z=\lim _{r \rightarrow 0^{+}}\left(\int_{\gamma_{11, r}(c)} f(z) d z+\int_{\gamma_{12, r}(c)} f(z) d z\right) .
$$

Definition 4 is consistent as $\gamma:[a, b] \rightarrow \mathbb{C}$ is a piecewise path. As expected, if an improper contour integral is convergent it converges to its principal value respect to the arc length but the latter may exist though the corresponding improper integral does not.

Definition 4 of Cauchy principal value contour integral of $f$ along $\gamma$ with respect to the arc length coincides with Definition 2 whenever the path $\gamma$ satisfies the following definition at the points where $f$ is unbounded.

Definition 5. We will say that a piecewise path $\gamma:[a, b] \rightarrow \mathbb{C}$ is locally isometric around $c$, where $a<c<b$, if there is some $r_{0}>0$ such that if $L(\gamma[u, c])=L(\gamma[c, v])<r_{0}$ with $a<u<c<v<b$, then $|\gamma(c)-\gamma(u)|=$ $|\gamma(c)-\gamma(v)|$. We will say that the path $\gamma$ is isometric if it is locally isometric around every point $c$ of $] a, b[$.

If $f$ is a function defined on a given piecewise path $\gamma:[a, b] \rightarrow \mathbb{C}$ except at finitely many points around which $\gamma$ is locally isometric, the Cauchy principal value contour integral of $f$ along $\gamma$ respect to the arc length coincides with PV $\int_{\gamma} f(z) d z$. In particular, as the path $\gamma(t)=t$ is isometric, Definition 4 generates the Cauchy improper real integral in the same way as Definition 2 does. Straight chords and arcs of circumference are examples of isometric paths.

\section{Cauchy Principal Value Contour Integral and Residue Theory}

Next, we will show how the principal value contour integrals may be calculated via the residue theorem whenever the contour $\gamma$ is closed and the singularities of $f$ along $\gamma$ are avoidable or poles of order 1. Firstly, let us introduce some notation. Given $\theta_{1}<\theta_{2}$ two real numbers with $\theta_{2}-\theta_{1} \leq 2 \pi$ and $z_{0} \in \mathbb{C}$, we represent the sector of vertex $z_{0}$ determined by $\theta_{1}$ and $\theta_{2}$ as

$$
C\left(z_{0}, \theta_{1}, \theta_{2}\right)=\left\{z \in \mathbb{C}: \theta_{1} \leq \arg \left(z-z_{0}\right) \leq \theta_{2}\right\} .
$$

Given $r>0$, we represent by $C_{r}\left(z_{0}, \theta_{1}, \theta_{2}\right)$ the arc of circumference of radius $r$ contained in $C\left(z_{0}, \theta_{1}, \theta_{2}\right)$, i.e., $C_{r}\left(z_{0}, \theta_{1}, \theta_{2}\right)=\left\{z_{0}+r e^{i t}: t \in\left[\theta_{1}, \theta_{2}\right]\right\}$.

We recall that $\lim _{z \rightarrow z_{0}} f(z)=k \in \mathbb{C}$ uniformly in $C\left(z_{0}, \theta_{1}, \theta_{2}\right)$ if, given $\varepsilon>0$, there exists some $\delta>0$ such that for all $0<r<\delta, \max \left\{|f(z)-k|: z \in C_{r}\left(z_{0}, \theta_{1}, \theta_{2}\right)\right\}<\varepsilon$.

For the sake of completeness, we include the proof of the following known result in which we recall that the first limit that appears corresponds to the residue of the function $f$ at a singular point $z_{0}$ of $f, \operatorname{Res}\left[f(z), z_{0}\right]$, whenever $z_{0}$ is a pole of order 1 , or an avoidable singular point of $f$.

Lemma 1. Let $f(z)$ be a function such that $\lim _{z \rightarrow z_{0}}\left(z-z_{0}\right) f(z)=k \in \mathbb{C}$ uniformly in $C\left(z_{0}, \theta_{1}, \theta_{2}\right)$, then $\lim _{r \rightarrow 0^{+}} \int_{C_{r}\left(z_{0}, \theta_{1}, \theta_{2}\right)} f(z) d z=i k\left(\theta_{2}-\theta_{1}\right)$. 
Proof 1. Given $\varepsilon>0$ there exists some $\delta>0$ so that for all $0<r<\delta$, $\max \left\{|g(z)|: z \in C_{r}\left(z_{0}, \theta_{1}, \theta_{2}\right)\right\}<\varepsilon$ where $g(z)=\left(z-z_{0}\right) f(z)-k$. Then

$$
\int_{C_{r}\left(z_{0}, \theta_{1}, \theta_{2}\right)} f(z) d z=\int_{C_{r}\left(z_{0}, \theta_{1}, \theta_{2}\right)} \frac{g(z)+k}{z-z_{0}} d z=\mathrm{i} \int_{\theta_{1}}^{\theta_{2}}\left(k+g\left(z_{0}+r e^{i t}\right) d t .\right.
$$

Hence $\left|\int_{C_{r}\left(z_{0}, \theta_{1}, \theta_{2}\right)} f(z) d z-\mathrm{i} k\left(\theta_{2}-\theta_{1}\right)\right| \leq \int_{\theta_{1}}^{\theta_{2}}\left|g\left(z_{0}+r e^{i t}\right)\right| d t<\varepsilon\left(\theta_{2}-\theta_{1}\right)$ for $r<\delta$.

Finally, let us recall that the derivative from the left (resp. right) of a real function $g$ at a point $c$ is given by the following limit whenever it does exist,

$$
g_{-}^{\prime}(c)=\lim _{x \rightarrow c^{-}} \frac{g(x)-g(c)}{x-c}\left(\text { resp. } g_{+}^{\prime}(c)=\lim _{x \rightarrow c^{+}} \frac{g(x)-g(c)}{x-c}\right) .
$$

The tangent ray at $c$ from the left (resp. right) is the half-line formed by the points

$$
\left.\left(c+t, g(c)+g_{-}^{\prime}(c) t\right), t \leq 0 \operatorname{resp} \cdot\left(c+t, g(c)+g_{+}^{\prime}(c) t\right), t \geq 0\right)
$$

We will denote its angle with $O X$ by $\alpha_{\mathrm{L}}(c)\left(=\tan ^{-1} g_{-}^{\prime}(c)\right)\left(\right.$ resp. $\left.\alpha_{\mathrm{R}}(c)\left(=\tan ^{-1} g_{+}^{\prime}(c)\right)\right)$. When there exists $g^{\prime}(c)$, both tangent rays at $c$ do exist, have the same direction and opposite sense conforming the tangent line to $g$ at $c$.

Now we are ready to prove a result that enables Cauchy principal value contour integral calculus via complex analysis residue theory. As usual, closed contours are always considered to be counterclockwise oriented.

Theorem 1. Let $D$ the region enclosed by a closed piecewise path $\gamma$. If a function $f$ is holomorphic on $\gamma$ and $D$, except at finitely many singular points $z_{1}, \ldots, z_{n} \in$ int $(D)$ and at finitely many poles $p_{1}, \ldots, p_{k}$ of order 1 which are located on $\gamma$, then

$$
\mathrm{PV} \int_{\gamma} f(z) d z=2 \pi \mathrm{i} \sum_{i=0}^{n} \operatorname{Res}\left[f(z), z_{i}\right]-\mathrm{i} \sum_{i=0}^{k}\left(\alpha_{R}\left(p_{i}\right)-\alpha_{L}\left(p_{i}\right)\right) \operatorname{Res}\left[f(z), p_{i}\right] .
$$

In particular, if $\gamma$ has tangent line on each pole $p_{i}$, then

$$
\mathrm{PV} \int_{\gamma} f(z) d z=2 \pi \mathrm{i} \sum_{i=0}^{n} \operatorname{Res}\left[f(z), z_{i}\right]+\pi \mathrm{i} \sum_{i=0}^{k} \operatorname{Res}\left[f(z), p_{i}\right] .
$$

Proof 2. Taking center in each pole $p_{i}, 1 \leq i \leq k$, we consider adequate $\operatorname{arcs}$ of circumference $C_{r}\left(p_{i}\right)$ of radius $r>0$ small enough so that they are pairwise disjoint, their ending points rely on $\gamma$, and that when we let $\gamma_{r}$ to be the closed piecewise path constructed by replacing the portions of $\gamma$ connecting the ending points of each $C_{r}\left(p_{i}\right), 1 \leq i \leq k$, by $C_{r}\left(p_{i}\right)$ itself, the region enclosed by $\gamma_{r}$ does include every point $z_{i}, 1 \leq i \leq n$, but not any of the $p_{1}, \ldots, p_{k}$.

Then the classical residue theorem assures that for such $r$ small enough,

$$
\int_{\gamma_{r}} f(z) d z=2 \pi \mathrm{i} \sum_{i=0}^{n} \operatorname{Res}\left[f(z), z_{i}\right]
$$

On the other hand

$$
\lim _{r \rightarrow 0^{+}} \int_{\gamma_{r}} f(z) d z=P V \int_{\gamma} f(z) d z+\lim _{r \rightarrow 0^{+}} \sum_{i=0}^{k} \int_{C_{r}\left(p_{i}\right)} f(z) d z .
$$


From (16) and Lemma 1, it follows that

$$
2 \pi \mathrm{i} \sum_{i=0}^{n} \operatorname{Res}\left[f(z), z_{i}\right]=P V \int_{\gamma} f(z) d z+\mathrm{i} \sum_{i=0}^{k}\left(\alpha_{R}\left(p_{i}\right)-\alpha_{L}\left(p_{i}\right)\right) \operatorname{Res}\left[f(z), p_{i}\right]
$$

Therefore, (14) holds.

Now, (15) is an immediate corollary of (14) since $\alpha_{R}\left(p_{i}\right)-\alpha_{L}\left(p_{i}\right)=-\pi$ at each pole $p_{i}$ in which $\gamma$ has tangent line.

Theorem 1 becomes the sibling of the method that evaluates Cauchy principal values of some improper real integrals as the sum of $2 \pi \mathrm{i}$ times the sum of residues of the function involved at the singular points located in the half-plane $\operatorname{Im}(z)>0$ plus $\pi$ i times the sum of residues at the poles of order 1 located on the real axis $\operatorname{Im}(z)=0$, cf. [16-18]. In fact, it is easy to prove that well-known result as consequence of Theorem 1.

\section{Applications}

Cauchy principal value integrals have continuously attracted a lot of attention, cf. [19-22]. In this section, we will take advantage of the Cauchy principal value contour introduced in the previous section and the tools associated with residue theory therein obtained in order to get the exact value of some relevant Cauchy principal values of real improper integrals.

\subsection{Quadrature of some Cauchy Principal Values}

In analysis courses the integral $I=\int_{0}^{2 \pi} R(\sin \alpha, \cos \alpha) d \alpha$ is transformed into a contour complex integral by considering $z=\exp (\mathrm{i} \alpha), \alpha \in[0,2 \pi]$, when $R(x, y)$ is a rational function. Then $I$ becomes $\int_{\gamma} R\left(\frac{z-z^{-1}}{2 \mathrm{i}}, \frac{z+z^{-1}}{2}\right) \frac{d z}{\mathrm{i} z}$, with $\gamma=C_{1}(0)$ the circumference of center 0 and radius 1 , which may be evaluated with residue theory whenever the integrand is an holomorphic function on $\gamma, \mathrm{cf}$. [16-18]. This applies, among others, to integrals of the type $\int_{0}^{2 \pi} \frac{\sin (n \alpha)}{a+b \cos \alpha} d \alpha$ if $|a|>|b|$ but not if $|a| \leq|b|$ since in the latter case the integral is improper and consequently the aforementioned transformation is not executed in analysis courses. However, when principal value is of interest, the techniques of this paper may help as the following example shows.

Example 2. Find the Cauchy principal value of $I=\int_{0}^{2 \pi} \frac{1}{\sqrt{3}-2 \cos \alpha} d \alpha$. This is an improper integral since the denominator becomes 0 at $\frac{\pi}{6}$ and $\frac{5 \pi}{6}$. In order to find the principal value we note that the change of variable $z=\exp (i \alpha)$ transforms the isometric straight segment $[0,2 \pi]$ into the isometric circumference $\gamma=C_{1}(0)$. Then, according to Theorem 1 ,

$$
\mathrm{PV} \int_{\gamma} \frac{1}{\sqrt{3}-2 \frac{z+z^{-1}}{2}} \frac{d z}{\mathrm{i} z}=-\mathrm{i} \mathrm{PV} \int_{\gamma} f(z) d z,
$$

where $f(z)=\frac{1}{z^{2}-\sqrt{3} z+1}$. Here $f$ has got poles of order 1 at $p_{1}=\exp \left(i \frac{\pi}{6}\right)$ and $p_{2}=\exp \left(i \frac{5 \pi}{6}\right)$, which lie on $\gamma$. Finding

$$
\operatorname{Res}\left[f(z), p_{1}\right]=\lim _{z \rightarrow p_{1}}\left(z-p_{1}\right) f(z)=-\mathrm{i}, \quad \operatorname{Res}\left[f(z), p_{2}\right]=\lim _{z \rightarrow p_{2}}\left(z-p_{2}\right) f(z)=\mathrm{i},
$$

Theorem 1 provides $P V(I)=0$.

\subsection{Some Relevant Integrals in Aerodynamics}

In aerodynamics, the thin airfoils theory leads to the Glauert's integral, [11,22-25],

$$
\mathrm{PV} \int_{0}^{\pi} \frac{\cos n x}{\cos x-\cos \theta} d x=\pi \frac{\sin (\mathrm{n} \theta)}{\sin \theta}, n \in \mathbb{N}, 0<\theta<\pi .
$$


There are several ways to approach this integral. For instance, Quori's proof [25] uses properties exhibited by the velocity components on the $x$-axis due to a source distribution on a strip of the same axis which are a particular case of a more general property of the functions of a complex variable and then applies a Zhukowsky transformation and two eliptic functions which are somehow not trivial to get the Glauert integral. Here we show that our Theorem 1 provides a straightforward proof.

Glauert's integral (4) is an improper integral as the denominator becomes 0 . Since the integrand is an even function, if we write $I=\int_{-\pi}^{\pi} \frac{\cos n x}{\cos x-\cos \theta} d x$ and $J=\int_{-\pi}^{\pi} \frac{\sin n x}{\cos x-\cos \theta} d x$, then the given Glauert's integral will be given by $\frac{1}{2} \operatorname{Re}(\operatorname{PV}(I+\mathrm{i} J))$.

As in Example 2, the change of variable $z=\exp (\mathrm{i} x)$ transforms the isometric straight segment $[-\pi, \pi]$ into the isometric circumference $\gamma=C_{1}(0)$. Hence

$$
\mathrm{PV}(I+\mathrm{i} J)=\mathrm{PV} \int_{\gamma} \frac{z^{n}}{\frac{z+z^{-1}}{2}-\cos \theta} \frac{d z}{\mathrm{i} z}=-2 \mathrm{iPV} \int_{\gamma} f(z) d z, \text { with } f(z)=\frac{z^{n}}{z^{2}-2(\cos \theta) z+1} .
$$

Here $f(z)$ has got poles of order 1 at $p_{1}=\exp (\mathrm{i} \theta), p_{2}=\exp (-\mathrm{i} \theta)$ which lie on $\gamma$. Finding the residues of $f$ at them,

$$
\operatorname{Res}\left[f(z), p_{1}\right]=\lim _{z \rightarrow p_{1}}\left(z-p_{1}\right) f(z)=\frac{\exp (\mathrm{in} \theta)}{2 \mathrm{i} \sin \theta}, \quad \operatorname{Res}\left[f(z), p_{2}\right]=\lim _{z \rightarrow p_{2}}\left(z-p_{2}\right) f(z)=\frac{\exp (-\mathrm{i} n \theta)}{-2 \mathrm{i} \sin \theta},
$$

From Theorem 1, it follows that $\mathrm{PV} \int_{\gamma} f(z) d z=\pi \mathrm{i} \frac{\exp (\mathrm{i} n \theta)-\exp (-\mathrm{in} \theta)}{2 \mathrm{i} \sin \theta}=\pi \mathrm{i} \frac{\sin (\mathrm{n} \theta)}{\sin \theta}$. A fortiori, the requested Glauert's integral follows

$$
\mathrm{PV} \int_{0}^{\pi} \frac{\cos n x}{\cos x-\cos \theta} d x=\operatorname{Re}\left(\frac{1}{2}(-2 \mathrm{i}) \pi \mathrm{i} \frac{\sin (\mathrm{n} \theta)}{\sin \theta}\right)=\pi \frac{\sin (\mathrm{n} \theta)}{\sin \theta}
$$

Another Glauer's integral related to the above is

$$
\mathrm{PV} \int_{0}^{\pi} \frac{\sin n x \sin x}{\cos x-\cos \theta} d x=-\pi \cos (n \theta), n \in \mathbb{N}, 0<\theta<\pi .
$$

Its value may be deduced from (21) and trigonometric properties. However, we will check that the results introduced in the previous section prove to be friendly also in this case. Here again, the integrand is an even function. Consequently, if we write

$$
I=\int_{-\pi}^{\pi} \frac{\cos n x \sin x}{\cos x-\cos \theta} d x, J=\int_{-\pi}^{\pi} \frac{\sin n x \sin x}{\cos x-\cos \theta} d x
$$

then (25) corresponds to the value given by $\frac{1}{2} \operatorname{Im}(\operatorname{PV}(I+\mathrm{i} J))$.

As in (21), the change of variable $z=\exp (\mathrm{i} x)$ transforms the isometric straight segment $[-\pi, \pi]$ into the isometric circumference $\gamma=C_{1}(0)$ and

$$
\mathrm{PV}(I+\mathrm{i} J)=\mathrm{PV} \int_{\gamma} \frac{z^{n} \frac{z-z^{-1}}{2 i}}{\frac{z+z^{-1}}{2}-\cos \theta} \frac{d z}{\mathrm{i} z}=-\mathrm{PV} \int_{\gamma} g(z) d z \text {, where } g(z)=\frac{z^{n+1}-z^{n-1}}{z^{2}-2(\cos \theta) z+1}
$$

Here $g(z)$ has got poles of order 1 at $p_{1}=\exp (i \theta)$ and $p_{2}=\exp (-\mathrm{i} \theta)$ which lie on $\gamma$. Finding the residues of $g$ at them,

$$
\begin{gathered}
R_{1}=\operatorname{Res}\left[g(z), p_{1}\right]=\lim _{z \rightarrow p_{1}}\left(z-p_{1}\right) g(z)=\frac{\exp (\mathrm{i}(n+1) \theta)-\exp (\mathrm{i}(n-1) \theta)}{\exp (\mathrm{i} \theta)-\exp (-\mathrm{i} \theta)} \\
R_{2}=\operatorname{Res}\left[g(z), p_{2}\right]=\lim _{z \rightarrow p_{2}}\left(z-p_{2}\right) g(z)=\frac{\exp (-\mathrm{i}(n+1) \theta)-\exp (-\mathrm{i}(n-1) \theta)}{\exp (-\mathrm{i} \theta)-\exp (\mathrm{i} \theta)} .
\end{gathered}
$$


From Theorem 1, trivial calculations provide that

$$
\mathrm{PV} \int_{\gamma} g(z) d z=\pi \mathrm{i}\left(R_{1}+R_{2}\right)=2 \pi \mathrm{i} \cos (n \theta)
$$

Hence $\mathrm{PV}(I+\mathrm{i} J)=-2 \pi \mathrm{i} \cos (n \theta)$ and (25) follows immediately.

\section{Discussion}

There are many reasons for the interest in Cauchy principal values, the main one probably being the fact that integral equations with Cauchy principal value integrals have shown to be an adequate tool for the modeling of many physical situations-such as acoustics, fluid mechanics, elasticity, fracture mechanics, electromagnetic scattering problems, and so on. In this context, we have tackled the issue of two natural formalizations of the concept of Cauchy principal value to Contour integrals of complex functions and studied paths on which they coincide. On the way, this extension has proven to be useful for the quadrature of some Cauchy principal value of improper real integrals.

\section{Conclusions}

In this paper, we have studied two main ways of extending the Cauchy principal value concept to contour integrals and have considered quite a general case, here called isometric paths, in which both of them coincide. Isometric paths have proven to be useful in the change of variables commonly used in order to transform some real definite integrals into contour integrals along the unit circle in the complex field and the ulterior use of residue theory. This method has enabled an easy calculus of Cauchy principal values that are relevant in aerodynamic study of a $2 \mathrm{D}$ wing section. We expect that other contour integrals or improper integrals used in entropic scenarios may find their principal value calculated following the methods depicted in this paper.

Acknowledgments: The authors are grateful to Aerospace Engineering Faculty at the School of Engineering Design in Universitat Politècnica de València.

Author Contributions: Matilde Legua and Luis M. Sánchez-Ruiz conceived the ideas and developed jointly the techniques here used; Matilde Legua and Luis M. Sánchez-Ruiz performed the complex examples and calculations; Luis M. Sánchez-Ruiz analyzed the data and contributed aeronautical thermodynamics materials and analysis; both authors tested the results; Matilde Legua wrote the draft paper; Luis M. Sánchez-Ruiz and Matilde Legua revised and approved the final manuscript.

Conflicts of Interest: The authors declare no conflict of interest.

\section{References}

1. Cover, T.M.; Thomas, J.A. Elements of Information Theory; Wiley: New York, NY, USA, 1991.

2. Reza, F.M. An Introduction to Information Theory; Dover Publications: New York, NY, USA, 1994.

3. Neumann, T. Bayesian inference featuring entropic priors. In Proceedings of the 27th International Workshop on Bayesian Inference and Maximum Entropy Methods in Science and Engineering, Albany, NY, USA, 8-13 July 2007; Volume 954, pp. 283-292.

4. Michalowicz, J.V.; Nichols, J.M.; Bucholtz, F. Handbook of Differential Entropy; CRC Press, Taylor \& Francis: New York, NY, USA, 2014.

5. Young, L.S. Entropy in Dynamical Systems. In Entropy; Greven, A., Keller, G., Warnecke, G., Eds.; Princeton University Press: Princeton, NJ, USA, 2003.

6. Galapon, E.A. Erratum: "The Cauchy principal value and the Hadamard finite part integral as values of absolutely convergent integrals" [J. Math. Phys. 57, 033502 (2016)]. J. Math. Phys. 2017, 58, 019901. [CrossRef]

7. Cohen, S.M.; Davies, K.T.R.; Davies, R.W.; Howard Lee, M. Principal-value integrals-Revisited. Can. J. Phys. 2005, 83, 565-575. [CrossRef]

8. Goldstein, S. On the vortex theory of screw propellers. Proc. R. Soc. Lond. Ser. A 1929, 123, 440-465. [CrossRef] 
9. Goldstein, S. On laminar boundary layer flow near a position of separation. J. Mech. Appl. Math. 1948, 1, 43-69. [CrossRef]

10. Chattot, J.J. Effects of blade tip modifications on wind turbine performance using vortex model. Comput. Fluids 2008, 38, 1405-1410. [CrossRef]

11. Yves-Marie Scolan, J.L. On some integrals of Glauert's type. Appl. Math. Lett. 2005, 18, 631-633. [CrossRef]

12. Apostol, T. Mathematical Analysis, 2nd ed.; 5th print.; Addison-Wesley: Reading, MA, USA, 1981.

13. Robinson, A.; Laurmann, J.A. Wing Theory; Cambridge University Press: London, UK, 2013.

14. Meseguer Ruiz, J.; Sanz Andrés, A. Aerodinámica Básica, 2nd ed.; Ibergaceta Publicaciones: Madrid, Spain, 2011. (In Spanish)

15. Kanwal, R.P. Linear Integral Equations. In Theory and Technique; Academic Press, Inc.: New York, NY, USA, 1971.

16. Levinson, N.; Redheffer, R.M. Complex Variables; McGraw-Hill: New York, NY, USA, 1988.

17. Brown, J.W.; Churchill, R.V. Complex Variables and Applications, 9th ed.; McGraw-Hill: New York, NY, USA, 2013.

18. Sánchez Ruiz, L.M.; Legua, M. Fundamentos de Variable Compleja y Aplicaciones, 3rd ed.; Universitat Politècnica de València: València, Spain, 2016. (In Spanish)

19. Diethdm, K.; Köhler, P. Asymptotic behaviour of fixed-order error constants of modified quadrature formulae for Cauchy principal value integrals. J. Inequal. Appl. 2000, 5, 167-190.

20. Capobianco, M.R.; Criscuolo, G. On quadrature for Cauchy principal value integrals of oscillatory functions. J. Comput. Appl. Math. 2003, 156, 471-486. [CrossRef]

21. Elliott, D.; Venturino, E. Sigmoidal transformations and the Euler-Maclaurin expansion for evaluating certain Hadamard finite-part integrals. Numer. Math. 1997, 77, 453-465. [CrossRef]

22. Ioakimidis, N.I. On the uniform convergence of Gaussian quadrature rules for Cauchy principal value integrals and their derivatives. Math. Comput. 1985, 44, 191-198. [CrossRef]

23. Li, H.; Chen, K. Gauss quadrature rules for Cauchy principal value integrals with wavelets. Appl. Math. Comput. 2007, 186, 357-364. [CrossRef]

24. Katz, J.; Plotkin, A. Low Speed Aerodynamics, 2nd ed.; Cambridge University Press: Cambridge, UK, 2002.

25. Quori, F. A new computation technique of Glauert's integrals. Appl. Math. Lett. 1995, 8, 7-9. [CrossRef]

(C) 2017 by the authors. Licensee MDPI, Basel, Switzerland. This article is an open access article distributed under the terms and conditions of the Creative Commons Attribution (CC BY) license (http:/ / creativecommons.org/licenses/by/4.0/). 